\title{
Carnets
}

Revue électronique d'études françaises de l'APEF

Première Série - 1 Numéro Spécial | 2009

Cultures littéraires : nouvelles performances \& développement

\section{L'œuvre en français de Milan Kundera ou les malentendus de l'ignorance}

\section{Luís Carlos Pimenta Gonçalves}

\section{(2) OpenEdition}

Journals

Édition électronique

URL : http://journals.openedition.org/carnets/3873

DOI : $10.4000 /$ carnets.3873

ISSN : 1646-7698

Éditeur

APEF

Édition imprimée

Date de publication : 1 juin 2009

Pagination : 233-242

Référence électronique

Luís Carlos Pimenta Gonçalves, «L'œuvre en français de Milan Kundera ou les malentendus de

l'ignorance », Carnets [En ligne], Première Série - 1 Numéro Spécial | 2009, mis en ligne le 16 juin 2018, consulté le 19 avril 2019. URL : http://journals.openedition.org/carnets/3873 ; DOI : 10.4000/ carnets.3873

Carnets est mis à disposition selon les termes de la licence Creative Commons - Atribution - Pas d'utilisation commerciale 4.0 International. 


\title{
L'CEUVRE EN FRANÇAIS DE MILAN KUNDERA \\ ou les malentendus de l'ignorance
}

\author{
LUÍS CARLOS PIMENTA GONÇALVES \\ Universidade Aberta \\ luispg@univ-ab.pt
}

\section{Résumé}

Nous nous proposons lors de cette communication d'évoquer la réception de l'œuvre en français ${ }^{1}$ de Kundera à travers quelques malentendus identitaires, linguistiques, idéologiques et esthétiques qu'elle génère.

\footnotetext{
${ }^{1}$ Nous désignerons par « œuvre en français » tous les opus, romans ou essais, rédigés directement en français et toutes les œuvres dont la traduction a été revue entièrement par Kundera. 


\section{Malentendus identitaires}

Une question qui est loin d'être anodine : Kundera est-il un écrivain français d'origine tchèque $^{2}$ ou un écrivain tchèque de nationalité française ${ }^{3}$. Est-il slave, originaire de l'Europe de l'Est, de l'Europe Centrale ou de la MittelEuropa? Détails identitaires qui peuvent paraître inintéressants au lecteur de prime abord, mais dont l'absence de clarification fonde des malentendus dont a souffert l'auteur. Ainsi dans l'essai intitulé «Die Weltliteratur » de son dernier ouvrage, Le Rideau, Kundera rapporte un de ces malentendus littéraires et géographiques :

vers la fin des années soixante-dix, j'ai reçu le manuscrit de la préface écrite pour un de mes romans par un éminent slaviste qui me mettait en perpétuelle comparaison (flatteuse, bien sûr, à l'époque personne ne me voulait de mal) avec Dostoïevski, Gogol, Bounine, Pasternak, Mandelstam, et avec les dissidents russes. Effrayé, j'en ai empêché la publication. Non que je ressentisse une antipathie pour ces grands Russes, au contraire, je les admirais tous, mais en leur compagnie je devenais un autre. Je me rappelle toujours l'étrange angoisse que ce texte m'a causée : ce déplacement dans un contexte qui n'était pas le mien, je le vivais comme une déportation.

On notera, outre le mal-être d'une identité littéraire confisquée, l'incise entre parenthèses : « à l'époque personne ne me voulait de mal » comme s'il était conscient en 2005 que l'époque de la reconnaissance et de la consécration littéraires unanimes était bien révolue deux décennies après.

\section{Malentendus idéologiques}

Un autre malentendu fréquent, né dès son premier roman traduit en France, est de nature idéologique. Ainsi, de La Plaisanterie, Sartre retiendra tout particulièrement la critique politique. Pour lui, l'auteur n'entend pas « désigner seulement l'innocente facétie du héros, mais l'ensemble d'un système où une gaminerie sans conséquence conduit inévitablement son auteur à la déportation ${ }^{4}$. A l'évidence, il s'agit également pour l'auteur de Les mains sales, de marquer sa rupture d'ancien compagnon de route avec Moscou. Cette

\footnotetext{
${ }^{2}$ C'est ainsi que le définit François Busnel dans Lire de mai 2005.

${ }^{3}$ Robert Lévesque le considère ainsi dans un article intitulé « Écrivain(s) de l'exil, du 01/08/2003, publié sur le site www.lelibraire.org : Le portail du livre au Québec. L'auteur pose le problème de l'identité de façon intéressante : «Kundera écrit en français, ce qu'il fait depuis 1981 (depuis sa variation sur Diderot, Jacques et son maître), est-il encore un écrivain tchèque ? Non. Est-il devenu un écrivain français ? Non plus. II est simplement Kundera, qui écrit en français là où il habite, où il mange, où il rêve. »

${ }^{4}$ SARTRE, Jean-Paul, Préface à Trois Générations, p. XIX, cité par Martin Rizek (2001).
} 
interprétation d'un roman antistalinien fera l'unanimité de la presse d'alors et sera privilégiée par l'éditeur Claude Gallimard, dont le prière d'insérer en quatrième de couverture de la première édition de 1968 de La plaisanterie présentera le roman en soulignant qu'il s'agit d'un «Roman idéologique par excellence ».

La préface de cette même édition, signée Aragon, associe anachroniquement le roman au désespoir né de la fin brutale du Printemps de Prague alors que l'édition tchèque date de 1967. Après cette première traduction, et pendant très longtemps, le roman kundérien va être associé au témoignage sous forme littéraire d'un dissident. II est vrai que le destin de Kundera est celui d'un intellectuel engagé, interdit de publication dans son pays, puis proscrit, mais il est vrai aussi que son oeuvre de fiction ne saurait se résumer à la trajectoire biographique de son auteur.

L'attribution du Prix Médicis étranger en 1973 pour La Vie est ailleurs, alors qu'il réside encore en Tchécoslovaquie, prime autant l'oeuvre que l'opposant. II sera pourtant autorisé par les autorités tchécoslovaques à venir recevoir le prix, regagnant Prague peu après. Le souvenir qu'en a Robbe-Grillet, créateur de ce Prix, est très diffus et inexact comme en témoigne un entretien accordé à Radio Prague en 2002.

C'est moi qui ai fait venir Kundera en France puisque je lui ai fait donner le prix Médicis qui était un prix que j'avais fondé et dans lequel j'avais une grande importance. [...] Et donc j'avais fait donner ce prix à un livre très connu de Kundera, dont j'ai oublié le titre. C'est pour recevoir ce prix qu'il est venu en France. Et il est resté. II en a profité pour ne pas retourner dans ce pays communiste qu'il haïssait.

Dans ce souvenir altéré par le temps perce un autre malentendu celui d'un Kundera anticommuniste détestant. Pourtant, déjà installé en France, Kundera insiste sur le fait qu'il n'est pas un émigré et qu'il n'a choisi la France que comme patrie littéraire, obtenant une autorisation du gouvernement tchèque lui permettant de vivre et d'enseigner en dehors de son pays. Kundera veut privilégier une lecture non-historique, voire apolitique de son œuvre pour éviter qu'elle ne soit associée à une banale critique d'un régime. II va même jusqu'à affirmer dans un entretien rapporté par Martin Rizek dans sa thèse Comment devient-on Kundera:

II est des commentateurs possédés par le démon de la simplification qui les assassinent les œuvres en leur surimposant une interprétation politique. Ceux-là ne s'intéressent aux écrivains dits « de l'Est » que dans la mesure où leurs œuvres sont interdites. (Rizek, 2001: 180) 
Dès 1990, avec la publication de L'Immortalité qui échappe à l'univers tchécoslovaque, certains lecteurs et critiques sont déçus et troublés de ne pas retrouver le décor habituel des romans de Kundera. Alors que le mur de Berlin vient à peine de tomber, l'animateur de radio et de télévision Michel Polack signe dans L'Événement du Jeudi, de janvier 1990 un retentissant et lapidaire: «Kundera, go home ». II conseille au romancier d'origine tchèque d'écrire dans sa langue natale plutôt que directement en français et de rentrer au pays. Objurgation dont on retrouve d'une certaine façon l'écho dans l'incipit de L'Ignorance, dix ans après: "Qu'est-ce que tu fais encore ici! », s'exclame Sylvie l'amie française d'Irena à la réfugiée tchèque.

\section{Malentendus linguistiques et littéraires}

Mais c'est quand, le romancier adoptera définitivement le français comme langue d'écriture que les critiques se feront encore plus virulentes et surgiront d'autres malentendus littéraires. Angelo Rinaldi, dans I'Express du 15/1/1998, résume assez bien une opinion courante dans certains milieux littéraires:

l'écrivain tchèque, si excellent dans sa langue maternelle et désormais rallié au français - qui n'en est pas à un outrage près - n'a pas apprécié les grincements des confrères dans leur accueil à La Lenteur. Son ouvrage suivant, il a donc préféré le publier d'abord en traduction à l'étranger, où il s'est procuré de quoi amortir les chocs.

De la même façon, le dernier roman de Kundera, L'Ignorance, pour contrer d'éventuels malentendus, se soumet d'abord à l'épreuve de l'étranger et est publié tout d'abord en Espagne en 2000, avant d'être finalement édité en France, dans sa langue originelle, en 2003. Avant cela, L'ignorance paraîtra dans de nombreux pays, dont le Portugal en 2001, devenant même un best-seller en Italie. Ce délai de l'édition française s'explique selon Michel Déon, cité par André Clavel, dans L'Express, 3/4/2003, par le fait que Kundera «est très sensible et facilement blessé. II est devenu la bête noire d'un petit cercle qui impose sa loi au milieu littéraire. Jouissant d'une reconnaissance internationale, il préfère publier ses livres dans des pays où ils seront examinés la tête un peu plus froide.»

Ce retard dans l'édition française du dernier roman contribue à alimenter le ressentiment qui ne fait que s'aggraver auprès de son lectorat français qui se sent exclu de la primeur du dernier ouvrage. Pour le journaliste Pierre Assouline, le dernier essai, Le rideau, confirme ce qu'il pensait à propos de ses romans français. De l'écrivain francotchèque, Assouline déclare perfidement dans son blogue La République des livres: "Depuis 
qu'il a décidé d'écrire en français, il est écrit mal et pense lourd." (12/04/2005) ${ }^{5}$. Kundera serait donc littérairement condamné à écrire en tchèque, assigné à résidence dans une langue au détriment d'une autre qu'il a pourtant choisie depuis bientôt quinze ans avec passion. Sauf à considérer que la majorité des lecteurs français et francophones lisent le tchèque, la connaissance que l'on a de l'oeuvre kundérienne depuis La Plaisanterie, originellement mal traduite en français et en anglais passe nécessairement par la traduction. Son premier éditeur anglais avait jugé bon de supprimer des passages entiers sur la musique et le folklore et d'ordonner différemment les chapitres ${ }^{6}$. On peut donc affirmer que la grande majorité des critiques français qui ont découvert et apprécié le Kundera tchèque l'ont lu soit dans de mauvaises traductions soit dans des traductions revues, voire en partie rédigées par Kundera. Dans le pire des cas, ils ont pu apprécier la réécriture de Marcel Aymonin de La Plaisanterie, de 1968, au mieux le même roman «entièrement révisée par Claude Courtot et l'auteur » dans sa « version définitive » comme l'affirme la page de garde de 1985. Les aléas de la traduction ont fait que Kundera est devenu très attentif aux traductions d'autant qu'elles participent à la diffusion du roman et à son universalité. C'est ce qu'il affirme dans le Rideau en parlant du romancier : " il s'ouvre au monde au-delà de sa langue nationale » (Kundera, 2005: 80).

La révision du texte traduit par Kundera ne va pas sans recréation du texte source comme si le romancier ne pouvait s'empêcher de réécrire son texte, non pour l'améliorer mais pour le rendre plus perceptible. Ainsi, certaines références culturelles disparaissent au profit d'autres jugées plus évocatrices pour un public français. Parlant de compositeurs qui ont puisé leur inspiration dans la musique traditionnelle, le texte tchèque de La Plaisanterie

\footnotetext{
${ }^{5}$ Ce genre de critique aura d'autant plus d'impact qu'elle n'est pas isolée, surtout lors de la publication de L'Identité. Dans L'Express, 3/4/2003, André Clavel dans un article intitulé "L'intransigeant amoureux de la France" parle de cette relation tendue entre une partie de la critique française et Kundera:

"Et, pendant qu'il orchestre ce second exil, il est en train de devenir un écrivain français, qui dit adieu à sa langue maternelle pour adopter celle de sa terre d'accueil. Comme Beckett, Cioran, lonesco. Premières gammes: deux essais, L'Art du roman et Les Testaments trahis. II y ramasse toutes ses obsessions, en dénonçant la perversion d'une époque où le moralisme épurateur a accouché d'un nouveau fléau, invisible, rampant - la dictature des consciences. Un moraliste, Kundera? Oui. Et qui va, au fil des années 1990, se lancer dans l'ultime aventure: écrire ses romans en français. Avec ce commentaire: «J'ai préféré ma liberté à mes racines. La langue tchèque m'appelle: reviens à la maison, voyou! Mais je n'obéis plus. Je veux rester avec la langue dont je suis éperdument amoureux.» Un sacré défi. Qu'il relève en épurant au maximum ses scénarios. Et en signant deux romans très courts, La Lenteur (nouvelle bombe contre les imposteurs de la religion cathodique) et $L$ 'ldentité, amère comédie sur le thème du malentendu amoureux.

C'est à ce moment-là que certains critiques, qui avaient auparavant encensé Kundera, sortent le napalm, dans L'Express, Libération, Le Canard enchaîné. L'écrivain, lui, est en train d'achever L'Ignorance. Mais il refuse que Gallimard le publie. Kundera le francophile tourne le dos à la France. En avril 2000, son livre est traduit en espagnol, chez Tusquets. La presse applaudit et les ventes explosent. Même ovation l'année suivante en Italie, où L'Ignoranza devient un best-seller, avant de paraître aux Etats-Unis et en Grande-Bretagne. On se demande alors si le roman sortira un jour en France, et Michel Déon, qui connaît bien Kundera, lui sert d'ambassadeur: «ll est très sensible et facilement blessé. II est devenu la bête noire d'un petit cercle qui impose sa loi au milieu littéraire. Jouissant d'une reconnaissance internationale, il préfère publier ses livres dans des pays où ils seront examinés la tête un peu plus froide.» Mais le romancier, qui travaille actuellement sur un essai, a enfin donné son feu vert: avec un premier tirage de 100000 exemplaires, L'Ignorance arrive cette semaine dans les librairies. Et ce sera encore un grand moment de l'aventure kundérienne."

${ }^{6}$ ELGRABLY, Jordan, "Conversations with Milan Kundera", Salmagundi, no73, Winter 1987, p. 18, cité par Rizek, 2001: 292.
} 
et sa première traduction mentionnent Stravinsky, Janacek, Bartok et Enesco tandis que la dernière traduction, bien que conservant le musicien russe, jugé sans doute suffisamment connu en France depuis la création parisienne du Sacre, indique les noms de compositeurs du groupe des Six, jugés sans doute plus évocateurs: Milhaud et Honegger. La première version de la traduction de La Plaisanterie qui inaugure la réception traductrice auprès du public français glisse très souvent d'un registre courant, parfois familier vers un registre fortement oralisé, voire argotique qui sonne parfois comme du Céline. En procédant à la révision définitive de la traduction, Kundera enlèvera les marques argotiques de la traduction originale.

Au début du roman, nous avons une narration d'un des personnages, la journaliste Helena. Dans la traduction de 1968, cela donne : “Ç'avait été au fond vachement comique la première fois que j'étais entrée dans son cabinet» qui devient dans la "version française définitive » (Kundera, 1968: 27), de 1985, un énoncé linguistiquement moins marqué : «Cela avait été au fond franchement comique la première fois que j'étais entrée dans son bureau» (Kundera, 2003: 41). Le truchement du passage d'une langue dans une autre, malgré ses difficultés et ses aléas, apparaît comme un pis-aller face à l'écriture dans une langue de portée universelle qui rend l'oeuvre directement accessible à des dizaines, voire des centaines de millions de lecteurs potentiels à travers le monde. Ainsi, depuis La vie est ailleurs, en 1973, les oeuvres rédigées en tchèque sont d'abord publiées dans leur traduction française. Toujours dans Le rideau, dans l'essai consacré à la Weltliteratur - la littérature mondiale chère à Goethe -, Kundera affirme de façon abrupte «personne ne connaîtrait Kafka aujourd'hui, personne, s'il avait été tchèque ». De fait, les hasards de l'Histoire après 1918 avaient fait d'un auteur de langue allemande un Tchécoslovaque.

À partir de son installation en France et donc dès son roman Le livre du rire et de l'oubli, interdit de publication en Tchécoslovaquie, séparé donc de ses premiers lecteurs tchèques, le romancier, selon ce qu'affirme Chvatik dans son essai sur Le monde romanesque de Milan Kundera, a commencé à écrire pour ses traducteurs, privilégiant une certaine univocité du langage qui éviterait de cette façon des malentendus langagiers.

II choisit dès lors toujours (consciemment ou inconsciemment), parmi la foule d'expressions possibles, celles dont les sens est le plus clair, le plus précis, de manière à exclure au maximum les erreurs de traduction. (Chvatik, 1995: 131)

Le passage du tchèque au français conserve intactes les principales caractéristiques formelles de l'écriture kundérienne que l'ont peut résumer en empruntant les mots employés par François Ricard dans sa postface à l'édition Folio de L'ignorance: «[...] le lecteur 
familier du Kundera tchèque se sent d'emblée chez lui en abordant le Kundera français : c'est la même «voix», la même «méthode», le même univers esthétique. »

À l'en croire, il n'y aurait donc pas rupture mais continuité entre ces deux versants de l'œuvre romanesque en une condensation de l'espace textuel de thèmes déjà présents dans la première partie de l'œuvre romanesque.

L'écrivain Guy Scarpetta, dans un article publié dans Le Monde diplomatique de mai 2003, "Jeux de l'exil et du hasard », abonde dans le même sens. Pour lui, il n'y aurait aucune rupture dans la manière/matière kundérienne depuis ses premiers romans.

Tout se passe, en somme, comme si Kundera écrivait, depuis le début, un même long roman - dont l'unité ne serait pas assurée, comme dans le cycle balzacien, par le retour des personnages mais par celui d'un même arsenal thématique, sans fin varié, réactivé, prolongé, développé, corrigé.

Dans le Rideau parlant des derniers films de Fellini après le consensuel Amarcord de 1973, Kundera remarque que le réalisateur ne correspondant plus à l'image que l'on se faisait de son œuvre dérange et agace le public et la critique par son «modernisme antimoderne ». Ce faisant, on peut légitimement penser à Kundera lui-même quand il évoque la liberté découverte par le cinéaste italien.

Au cours de ces années, irrités à la fois par son esthétique très exigeante et par le regard désenchanté qu'il pose sur leur monde contemporain, les salons, la presse, le public (et même les producteurs) se détournent de lui ; ne devant plus rien à personne, il savoure la «joyeuse irresponsabilité » (je le cite) d'une liberté que, jusqu'alors, il n'a pas connue. (Kundera, 2005: 175)

\section{Les malentendus du paradoxe et de l'ironie}

La tentative de démystification du réel chez Kundera procède par paradoxe, ce qui contribue sans doute à alimenter d'autres malentendus, en s'attaquant aux opinions communes, à la doxa et en donnant une importance extrême à ce qui semble anecdotique. II donne à la notion de dissidence, uniquement cantonnée à la sphère de l'intellectuel pourchassée par un régime communiste, dans La Plaisanterie, dans Le livre du rire et de l'oubli ou dans L'insoutenable légèreté de l'être, une dimension bien plus vaste. Dans L'immortalité, la propagande du régime politique est substituée par la publicité aussi aliénante du système économique en proposant une critique de l'image qu'il appelle « imagologie » : «M'objecterez-vous que publicité et propagande n'ont pas de rapport entre 
elles, l'une étant au service du marché et l'autre de l'idéologie ? » (Kundera, 1990: 171). II est symptomatique que quelques années plus tard dans L'identité, le grand publicitaire brillant est un ancien trotskiste. Les idées deviennent des marques, la promotion d'un produit vaut celle d'une idée. Que l'on songe au «penser différemment » et à la référence à Orwell dans un célèbre spot publicitaire pour promouvoir l'ordinateur à la Pomme dans les années 80 .

Les vérités toutes faites, les grands principes de la Tchécoslovaquie communiste ridiculisés par Kundera hier, sont remplacés par une critique du grand mythe fondateur de la République française, La Déclaration des Droits de l'Homme.

Ainsi, grâce à Soljenitsyne, l'expression « droits de l'homme » a-t-elle retrouvé sa place dans le vocabulaire de notre temps ; je ne connais pas un homme politique qui n'invoque dix fois par jour « la lutte pour les droits de l'homme » ou « les droits de I'homme qu'on a bafoués.

Ce faisant, Kundera ironise-t-il sur un discours lénifiant de l'Occident et en passant s'attaque-t-il à une figure tutélaire de la dissidence. Les cliché et le stéréotype culturel sont également mis à mal dans le roman kundérien, la Suisse ainsi ne peut qu'être alpestre et calme, l'américain est un homme d'action écervelé, les Italiens bruyants et gesticulants. II pourfend également la gloire et la postérité littéraires. Hemingway et sa vision d'épouvante de sa survie littéraire : « tous mes amis étaient là et racontaient tous les cancans » et plus inquiétant encore, toujours dans ce même passage de L'immortalité: "dans toutes les universités d'Amérique une armée de professeurs classaient tout cela, l'analysaient, le développaient, fabriquant des milliers d'articles et des centaines de livres».

En guise de conclusion, nous pourrions dire que le changement dans l'œuvre kundérienne, critiqué par certains journalistes et lecteurs, est le fait d'une épure, d'une simplification induite par un changement de destinataire. Le projet est énoncé d'une certaine façon par un personnage du Le livre du rire et de l'oubli. L'exilée Tamina pour s'adapter à la France et à ses habitants doit reconstruire sa biographie, la «soulager » de ce qu'elle peut avoir d'obscur et d'incompréhensible pour des Français. Elle «était convaincue que si elle voulait que les gens d'ici comprennent quelque chose à sa vie, il fallait qu'elle la simplifie » (Rizek, 2001: 290).

Avec ses romans écrits directement en français, Kundera peut renouer avec le plaisir des mots, jongler avec la fonction poétique du langage et la faconde des slogans publicitaires dans L'Identité ou la crudité de termes obscènes dans La lenteur.

Quand l'entomologiste Berck, dans ce dernier roman, lors d'un colloque conspue une admiratrice trop envahissante et que celle-ci le somme de s'expliquer : «Pourquoi me dis-tu 
tout cela ? Pourquoi ? Comment dois-je le comprendre ?» II répond :« Tu dois le comprendre tel que je le dis! Au premier degré ! Au tout premier degré ! Poufiasse comme poufiasse, emmerdeuse comme emmerdeuse, cauchemar comme cauchemar, urine comme urine! » (Kundera, 1995: 100).

Au-delà de l'énormité provocatrice d'une telle déclaration, il faut y voir le caractère jubilatoire d'un discours transparent, univoque, immanent, libéré de la pesanteur de la traduction, vulgaire et brutal s'il le désire et faisant un pied de nez à la critique bien pensante. 


\section{Bibliographie}

ChVATIK, Kvetoslav (1995), Le monde romanesque de Milan Kundera, trad. Bernard Lortholary, Paris, Gallimard.

Kundera, Milan (1968), La Plaisanterie, trad. Marcel Aymonin, Paris, Gallimard.

KUNDERA (1990), Milan, L'immortalité, trad. Eva Bloch, postface François Ricard, Paris, Gallimard, coll. Folio.

KUndera, Milan (1995), La lenteur, postface de François Ricard, Paris, Gallimard, coll. Folio.

KUnderA, Milan, (2003), La Plaisanterie, trad. Marcel Aymonin, entièrement révisée par Claude Courtout et l'auteur, version définitive, postface de François Ricard, Paris, Gallimard, coll. Folio.

Kundera (2005), Milan, Le rideau, Paris, Gallimard, coll. Folio.

RIzeK, Martin (2001), Comment devient-on Kundera? Images de l'écrivain, écrivain de l'image, Paris, L'Harmattan. 